\title{
Preclinical Studies of Chemotherapy Using Histone Deacetylase Inhibitors in Endometrial Cancer
}

\author{
Noriyuki Takai and Hisashi Narahara \\ Department of Obstetrics and Gynecology, Faculty of Medicine, Oita University, Hasama-machi, Yufu-shi, Oita 879-5593, Japan \\ Correspondence should be addressed to Noriyuki Takai, takai@med.oita-u.ac.jp
}

Received 15 September 2009; Revised 23 November 2009; Accepted 14 January 2010

Academic Editor: Paul J. Hoskins

Copyright ( $) 2010$ N. Takai and H. Narahara. This is an open access article distributed under the Creative Commons Attribution License, which permits unrestricted use, distribution, and reproduction in any medium, provided the original work is properly cited.

\begin{abstract}
Because epigenetic alterations are believed to be involved in the repression of tumor suppressor genes and promotion of tumorigenesis in endometrial cancers, novel compounds endowed with a histone deacetylase (HDAC) inhibitory activity are an attractive therapeutic approach. In this review, we discuss the biologic and therapeutic effects of HDAC inhibitors (HDACIs) in treating endometrial cancer. HDACIs were able to mediate inhibition of cell growth, cell cycle arrest, apoptosis, and the expression of genes related to the malignant phenotype in a variety of endometrial cancer cell lines. Furthermore, HDACIs were able to induce the accumulation of acetylated histones in the chromatin of the $\mathrm{p} 21^{\mathrm{WAF} 1}$ gene in human endometrial carcinoma cells. In xenograft models, some HDACIs have demonstrated antitumor activity with only few side effects. In this review, we discuss the biologic and therapeutic effects of HDACIs in treating endometrial cancer, with a special focus on preclinical studies.
\end{abstract}

\section{Introduction}

Endometrial cancer is the seventh most common malignancy among women worldwide. Despite the fact that most cases are diagnosed at an early stage, the death rate has increased steadily over the past 20 years. The lack of an effective, standardized adjuvant treatment for women at a high risk of recurrence has contributed to these disappointing results (reviewed in [1]). The most frequent genetic alteration in type I endometrioid carcinoma is PTEN inactivation by mutation, followed by microsatellite instability and mutations of K-ras and $\beta$-catenin. In type II cancers, p53 mutation is the most frequent genetic alteration, followed by inactivation of p16 and e-cadherin and amplification of Her2/neu (reviewed in [1]).

One of the most important mechanisms in chromatin remodeling is the post-translational modification of the $\mathrm{N}$ terminal tails of histones by acetylation, which contributes to a "histone code" determining the activity of target genes [2]. Transcriptionally silent chromatin is composed of nucleosomes in which the histones have low levels of acetylation on the lysine residues of their amino-terminal tails. Acetylation of histone proteins neutralizes the positive charge on lysine residues and disrupts the nucleosome structure, allowing unfolding of the associated DNA with subsequent access by transcription factors, resulting in changes in gene expression. Acetylation of core nucleosomal histones is regulated by the opposing activities of histone acetyltransferases (HATs) and histone deacetylases (HDACs). HDACs catalyze the removal of acetyl groups on the amino-terminal lysine residues of core nucleosomal histones, and this activity is generally associated with transcriptional repression. HDACs remove the acetyl groups which then induce a positive charge on the histones, and this suppresses gene transcription, including tumor suppressor genes silenced in cancer. Moreover, acetylation of histones facilitates destabilization of DNAnucleosome interaction and renders DNA more accessible to transcription factors [3]. Aberrant recruitment of HDAC activity has been associated with the development of certain human cancers [4]. HDAC inhibitors (HDACIs) can inhibit cancer cell growth in vitro and in vivo, revert oncogenetransformed cell morphology, induce apoptosis, and enhance cell differentiation.

The classes of HDACIs that have been identified are: (a) organic hydroxamic acids (e.g., Trichostatin A (TSA) and suberoylanilide bishydroxamine (SAHA)) (b) short-chain 
TABLE 1: Overview of frequently used histone deacetylase inhibitors that are available for clinical and research purposes.

\begin{tabular}{|c|c|c|}
\hline Substance groups & Derivatives & Isotype \\
\hline \multirow{14}{*}{ Hydroxamates } & Trichostatin A (TSA) & I, II \\
\hline & Suberoylanilide hydroxamic acid (SAHA, vorinostat) & I, II, IV \\
\hline & LBH589 (panobinostat) & I, II, IV \\
\hline & PCI24781 (CRA-024781) & I, IIb \\
\hline & LAQ824 & I, II \\
\hline & PXD101 (belinostat) & I, II, IV \\
\hline & ITF2357 & I, II \\
\hline & SB939 & Unknown \\
\hline & JNJ-16241199 (R306465) & I \\
\hline & m-carboxycinnamic acid bishydroxamide (CBHA) & \\
\hline & Scriptaid & \\
\hline & Oxamflatin & \\
\hline & Pyroxamide & \\
\hline & Cyclic hydroxamic acid containing peptides (CHAPs) & \\
\hline \multirow{4}{*}{ Short chain fatty acids } & Butyrate & I, IIa \\
\hline & Valproate & I, IIa \\
\hline & AN-9 & \\
\hline & OSU-HDAC42 & \\
\hline \multirow{5}{*}{ Benzamides } & MS-275 (entinostat) & $1,2,3,9$ \\
\hline & MGCD0103 & $1,2,3,11$ \\
\hline & Pimelic diphenylamide & $1,2,3$ \\
\hline & M344 & \\
\hline & N-acetyldinaline (CI-994) & \\
\hline \multirow{5}{*}{ Cyclic tetrapeptides } & Apicidine & I, II \\
\hline & Trapoxins & \\
\hline & HC-toxin & \\
\hline & Chlamydocin & \\
\hline & Depsipeptide (FR901228 or FK228) (romidepsin) & $1,2,4,6$ \\
\hline Sulfonamide anilides & N-2-aminophenyl-3-[4-(4-methylbenzenesulfonylamino)-phenyl]-2-propenamide & \\
\hline \multirow{3}{*}{ Others } & Depudecin & \\
\hline & NDH-51 & \\
\hline & KD5150 & Pan-HDACI \\
\hline
\end{tabular}

Class I: HDAC1, -2, -3 and -8; class IIa: HDAC4, -5, -7, and -9; class IIb: HDAC 6, and -10; class III: SIRT1-7; class IV: HDAC11.

fatty acids (e.g., butyrates and valproic acid (VPA)), (c) benzamides (e.g., MS-275), (d) cyclic tetrapeptides (e.g., trapoxin), and (e) sulfonamide anilides [5] (see Table 1).

In this review, we discuss the biologic and therapeutic effects of HDACIs in treating endometrial cancer, with a special focus on preclinical studies.

\section{Mechanism of Action}

Histone deacetylases (HDACs) comprise a family of 18 genes that are subdivided into four classes [6]. Classes I, II, and IV are referred to as "classical" HDACs and are generally simultaneously targeted by most HDACIs (Table 1). HDACIs were initially discovered on the basis of their ability to reverse the malignant phenotype of transformed cells in culture. It has been shown that HDACIs carry the potential to activate differentiation programs on one hand, while on the other hand they were also shown to inhibit cell proliferation by cell cycle arrest in the G1 and/or G2 phases of the cell cycle and to induce apoptosis in cultured transformed cells. $\mathrm{p} 21^{\mathrm{WAF} 1}$ and $\mathrm{p} 27^{\mathrm{KIP} 1}$ are cyclin-dependent kinase inhibitors (CDKIs) that bind to cyclin-dependent kinase complexes and decrease kinase activity, and may act as key regulators of the G0/G1 accumulation (reviewed in [7]). The p $21^{\text {WAF1 }}$ expression in particular is induced by HDACIs in various cell lines. Additionally, this event is associated with both an increase in histone acetylation in the promoter region of the $\mathrm{p} 21^{\mathrm{WAF} 1}$ gene and a selective loss of a specific HDAC enzyme, HDAC1, in the same region [8]. Therefore, the upregulation of $\mathrm{p} 21^{\mathrm{WAF} 1}$ is a direct consequence of HDACIs on $\mathrm{p} 21^{\mathrm{WAF} 1}$ transcription. In the future, testing should be conducted using $\mathrm{p} 21^{\mathrm{WAF} 1}$-negative cell lines to see if $\mathrm{p} 21^{\mathrm{WAF} 1}$ is absolutely required for HDACI-induced growth arrest. 
Takai et al. examined the effect of HDACIs on the expression of $\mathrm{p} 21^{\mathrm{WAF} 1}$ and $\mathrm{p} 27^{\mathrm{KIP} 1}$ in endometrial cancer cells by Western blot analysis. HDACIs markedly upregulated the level of $\mathrm{p} 21^{\mathrm{WAF} 1}$ and $\mathrm{p} 27^{\mathrm{KIP} 1}$ proteins, which were expressed at negligible levels in the untreated cell lines. Conversely, HDACIs decreased the levels of cyclin D1 and cyclin D2. HDACIs decreased the bcl-2 levels. E-cadherin binds to $\beta$ catenin and can act as a tumor suppressor gene; its promoter has $\mathrm{CpG}$ islands which are frequently methylated in selected cancers. HDACIs markedly increased the expression level of E-cadherin in endometrial cancer cells and exhibited antiproliferative activity in these cells [9-14]. HDACIs have also been shown to generate reactive oxygen species (ROS) in solid tumor and leukemia cells [15-17], which may contribute to the mechanism. HDACIs have been shown to inhibit angiogenesis. HDACIs repress the expression of proangiogenic factors such as HIF1 $\alpha$, VEGF, VEGF receptor, endothelial nitric oxide synthase, IL-2 and IL-8 and the induction of antiangiogenic factors, such as p53 and von Hippel-Lindau (reviewed in [18]). HDACIs should not be considered to act solely as enzyme inhibitors of HDACs. A large variety of nonhistone transcription factors and transcriptional co-regulators are known to be modified by acetylation. HDACIs can alter the degree of acetylation nonhistone effector molecules and thereby increase or repress the transcription of genes by this mechanism. Examples include: ACTR, cMyb, E2F1, EKLF, FEN 1, GATA, HNF-4, HSP90, Ku70, NFkB, PCNA, p53, RB, Runx, SF1 Sp3, STAT, TFIIE, TCF, YY1, and so forth, (reviewed in [18]).

\section{Overview and Preclinical Studies of HDACIs}

A variety of structurally distinct classes of compounds that inhibit deacetylation of both histone and non-histone proteins have gradually been identified (Table 1 ). Despite the shared capacity of each class of HDACIs to promote histone acetylation, individual HDACIs exert different actions on signal transduction and the induction of differentiation and/or apoptosis. Table 2 shows data from different reports investigating endometrial cancer cell lines treated with different classes of HDACIs. Many of the in vitro studies use the Ishikawa cell line. Nishida. succeeded in establishing of a well-differentiated endometrial adenocarcinoma cell line, Ishikawa cells, from a 39-year old Japanese patient more than 20 years ago [19]. Because this cell line bears estrogen and progesterone receptors, the cells have been used in numerous basic research areas such as reproductive biology and molecular science.

3.1. Trichostatin A (TSA). The trichostatins were initially isolated from Streptomyces hygroscopicus as antifungal antibiotics in 1976 [20, 21]. About 10 years later, TSA and its analogues were discovered to induce cell differentiation of murine erythroleukemia cells and to induce hyperacetylation of histone proteins at nanomolar concentrations. TSA has been extensively studied; it has antitumor activity and can induce differentiation of some cancer cell lines, but its
TABle 2: Data investigating endometrial cancer cell lines treated with different classes of HDACIs.

\begin{tabular}{|c|c|c|}
\hline HDACI & Cell line & ED50 (M) \\
\hline \multirow{7}{*}{ TSA } & Ishikawa & $5.2 \times 10^{-8}$ \\
\hline & HEC-1B & $5.1 \times 10^{-8}$ \\
\hline & HEC59 & $7.0 \times 10^{-8}$ \\
\hline & RL95-2 & $9.8 \times 10^{-8}$ \\
\hline & KLE & $7.2 \times 10^{-8}$ \\
\hline & AN3CA & $1.9 \times 10^{-8}$ \\
\hline & Ark2 & $2.5 \times 10^{-8}$ \\
\hline \multirow{6}{*}{ SAHA } & Ishikawa & $7.8 \times 10^{-7}$ \\
\hline & HEC-1B & $7.8 \times 10^{-7}$ \\
\hline & HEC59 & $1.2 \times 10^{-6}$ \\
\hline & RL95-2 & $2.4 \times 10^{-6}$ \\
\hline & KLE & $2.5 \times 10^{-6}$ \\
\hline & AN3CA & $3.1 \times 10^{-6}$ \\
\hline \multirow{3}{*}{ CBHA } & Ishikawa & $1.8 \times 10^{-6}$ \\
\hline & HHUA & $2.5 \times 10^{-6}$ \\
\hline & HEC-1B & $2.2 \times 10^{-6}$ \\
\hline Scriptaid & Ishikawa & $9.0 \times 10^{-6}$ \\
\hline \multirow{6}{*}{$\mathrm{NaB}$} & Ishikawa & $8.3 \times 10^{-4}$ \\
\hline & HEC-1B & $8.4 \times 10^{-4}$ \\
\hline & HEC59 & $1.8 \times 10^{-3}$ \\
\hline & RL95-2 & $3.0 \times 10^{-3}$ \\
\hline & KLE & $3.9 \times 10^{-3}$ \\
\hline & AN3CA & $4.1 \times 10^{-3}$ \\
\hline \multirow{6}{*}{ VPA } & Ishikawa & $7.0 \times 10^{-4}$ \\
\hline & HEC-1B & $7.5 \times 10^{-4}$ \\
\hline & HEC59 & $8.2 \times 10^{-4}$ \\
\hline & RL95-2 & $2.5 \times 10^{-3}$ \\
\hline & KLE & $2.3 \times 10^{-3}$ \\
\hline & AN3CA & $3.8 \times 10^{-3}$ \\
\hline \multirow{6}{*}{ MS-275 } & Ishikawa & $9.7 \times 10^{-7}$ \\
\hline & HEC-1B & $2.2 \times 10^{-6}$ \\
\hline & RL95-2 & $1.0 \times 10^{-6}$ \\
\hline & HHUA & $7.8 \times 10^{-7}$ \\
\hline & AN3CA & $5.0 \times 10^{-7}$ \\
\hline & Ark2 & $5.0 \times 10^{-7}$ \\
\hline M344 & Ishikawa & $2.3 \times 10^{-6}$ \\
\hline Apicidine & Ishikawa & $1.0 \times 10^{-6}$ \\
\hline PsA & Ishikawa & $7.5 \times 10^{-6}$ \\
\hline \multirow{3}{*}{ Oxamflatin } & Ishikawa & $2.5 \times 10^{-7}$ \\
\hline & AN3CA & $2.5 \times 10^{-7}$ \\
\hline & Ark2 & $2.5 \times 10^{-7}$ \\
\hline
\end{tabular}

clinical utility has been restricted because of toxic sideeffects in vivo [22]. TSA causes mitotic arrest through the formation of aberrant mitotic spindles, probably by interfering with chromosome attachment, but does not affect mitotic microtubules [22]. This effect may account for the higher cytotoxicity of TSA in comparison to other HDACIs 
(i.e., suberoylanilide bishydroxamine). HDAC inhibition is not believed to have a generalized effect on the genome, but only on the transcription of a small subset of the genome. Differential display analysis of transformed lymphoid cell lines revealed that the expression of only $2 \%-5 \%$ of transcribed genes is changed significantly after treatment with TSA [23]. The effective dose of TSA that inhibited 50\% clonal growth (ED50) of the endometrial cancer cell lines (Ishikawa, HEC-1B, HEC59, RL95-2, KLE, and AN3CA) was calculated, and ranged between $5.1 \times 10^{-8} \mathrm{M}$ and 1.9 $\times 10^{-7} \mathrm{M}$ [9] (Table 2). Dowdy et al. demonstrated that combined treatment with TSA and paclitaxel caused synergistic inhibition of cell growth of Ark2 and KLE endometrial cancer cells [24]. These effects were confirmed in a mouse xenograft model. Treatment with TSA and paclitaxel led to a significant increase in acetylated tubulin and microtubule stabilization. This study provides the evidence of nonhistone protein acetylation as one possible mechanism by which HDACIs reduce cancer growth.

\subsection{Suberoylanilide Bishydroxamine (SAHA, Vorinostat).} Hydroxamic acid type inhibitors make up the largest and broadest group of HDACIs described to date. The inhibition of HDACs by SAHA occurs through a direct interaction with the catalytic site of the enzyme, as shown by X-ray crystallography studies [25]. Among the synthetic HDACIs, SAHA is the most advanced candidate as a cancer therapeutic drug, and is under phase I and II clinical trials [26, 27]. SAHA has significant antitumor activity against many tumor types at dosages that can be tolerated by patients when administered intravenally and orally [28]. Some HDACIs (e.g., TSA and trapoxin) are of limited therapeutic use due to poor bioavailability in vivo and have toxic side effects at high doses. SAHA, however, is relatively safe and non-toxic in vivo. The effective dose of SAHA that inhibited 50\% clonal growth (ED50) of the endometrial cancer cell lines (Ishikawa, HEC-1B, HEC59, RL95-2, KLE, and AN3CA) was calculated and ranged between $7.8 \times 10^{-7} \mathrm{M}$ and $3.1 \times 10^{-6} \mathrm{M}$ [9] (Table 2).

3.3. m-carboxycinnamic Acid Bishydroxamide (CBHA). CBHA is a member of a recently synthesized family of hybrid polar compounds that have been shown to be inhibitors of HDAC [29] and potent inducers of transformed cell growth arrest and terminal differentiation at micromolar (LD50 range, $1-4 \mu \mathrm{M}$ ) concentrations [30]. The effective dose of CBHA that inhibited $50 \%$ clonal growth (ED50) of the endometrial cancer cell lines (Ishikawa, HEC-1B, HHUA) was calculated and ranged between $1.8 \times 10^{-6} \mathrm{M}$ and $2.5 \times$ $10^{-6} \mathrm{M}$ for CBHA [10] (Table 2). On the other hand, normal endometrial epithelial cells were viable after treatment with the same doses of CBHA that induced growth inhibition of endometrial cancer cells [10].

3.4. Scriptaid. Using a high-throughput system based on a stably integrated transcriptional reporter to screen a library of 16,320 compounds (DIVERset, Chembridge, San Diego, CA), Su et al. identified a novel HDACI, termed Scriptaid
[31]. Nullscript, which possesses a shorter side-chain (3C) than Scriptaid $(5 \mathrm{C})$ between the tricyclic core and the carbonyl group, was inactive in transcriptional facilitation. This confirms that the linker chain has to be a certain length for HDAC inhibition to occur. Scriptaid has a common structure with TSA and SAHA, that is, a hydroxamic acid zinc-binding group linked via a spacer (5 or $6 \mathrm{CH} 2$ ) to a hydrophobic group [31]. Using an immunoblotting assay of histone deacetylation, Su et al. demonstrated that Scriptaid is a potent HDACI with a $>100$-fold increase in histone acetylation, with relatively low toxicity [31]. Scriptaid conferred the greatest effect on augmentation of the signal transduction TGF $\beta$ pathway, including a number of human suppressor genes such as SMAD4 [31]. The effect of Scriptaid in human cancers, however, has not been fully examined. A recent study by Keen et al. indicated that Scriptaid had a significant growth-suppressing effect on ERnegative human breast cancer cells [32]. The effective dose of Scriptaid that inhibited 50\% clonal growth (ED50) of the Ishikawa endometrial cancer cell line was calculated at $9.0 \times 10^{-6} \mathrm{M}$ [11] (Table 2). On the other hand, normal endometrial epithelial cells were viable after treatment with the same doses of Scriptaid that induced growth inhibition of endometrial cancer cells [11].

3.5. Sodium Butyrate (NaB). It was first reported in 1978 that millimolar concentrations of sodium butyrate $(\mathrm{NaB})$ inhibited HDACs in vitro [33]. $\mathrm{NaB}$ is normally present in the human colon as a product of the metabolic degradation of complex carbohydrates by colonic bacteria and regulates the physiological differentiation of colonocytes, suggesting its possible use in the prevention of colorectal cancer and the treatment of premalignant and neoplastic lesions. Butyrate and its derivatives have a long history of safe clinical use in the treatment of inherited and acquired metabolic disorders. Some studies suggest that many of the cellular activities of phenylbutyrate are more dependent on its butyric acid component than its phenyl group. A recent study by Terao et al. indicated that $\mathrm{NaB}$ had a significant growthsuppressing effect on human endometrial and ovarian cancer cells irrespective of their p53 gene status [34]. NaB, a low-potency HDACI, has been extensively studied; it has antitumor activity and can induce differentiation of some cancer cell lines, but its clinical utility has been restricted by its short half-life ( 5 minutes), limiting the ability to achieve a therapeutic plasma level. $\mathrm{NaB}$ and phenylbutyrate are degraded rapidly after i.v. administration and therefore require high doses exceeding $400 \mathrm{mg} / \mathrm{kg} /$ day [35]. Furthermore, these compounds are not specific for HDACs as they also inhibit phosphorylation and methylation of proteins as well as DNA methylation [35]. The effective dose of $\mathrm{NaB}$ that inhibited 50\% clonal growth (ED50) of the endometrial cancer cell lines (Ishikawa, HEC-1B, HEC59, RL95-2, KLE, and $\mathrm{AN} 3 \mathrm{CA}$ ) has been calculated and ranged between $8.3 \times$ $10^{-4} \mathrm{M}$ and $4.1 \times 10^{-3} \mathrm{M}$ for $\mathrm{NaB}$ [9] (Table 2).

3.6. Valproic Acid (VPA). Valproic acid, a shortchain fatty acid, has been approved for clinical use in the treatment of epilepsy and is frequently used in clinical trials and for in 
vitro research based on its HDAC inhibitory effect at comparatively high (millimolar) concentrations [36]. Valproic acid has also been identified as an antiproliferative agent and HDACI [37]. Some HDACIs (e.g., TSA and trapoxin) are of limited therapeutic use due to poor bioavailability in vivo as well as toxic side effects at high doses, but VPA is relatively safe and non-toxic in vivo. The effective dose of VPA that inhibited 50\% clonal growth (ED50) of the endometrial cancer cell lines (Ishikawa, HEC-1B, HEC59, RL95-2, KLE, and AN3CA) has been calculated and ranged between $7.0 \times$ $10^{-4} \mathrm{M}$ and $3.8 \times 10^{-3} \mathrm{M}$ [9] (Table 2).

A previous study tested the ability of VPA to inhibit the growth of human HEC-1B endometrial cancer tumors growing in immunodeficient mice [9]. Administration of VPA remarkably suppressed the growth of the tumors ( $P$ $<.01)$. No significant differences in either the mean weights, histology of the internal organs, and mean blood chemistries, including liver parameters and hematopoietic values, were found between diluent-treated mice and those that received 5 weeks of therapy. These tumors were sampled for the expression of $\mathrm{p} 21^{\mathrm{WAF} 1}$ using immunohistochemistry on formalinfixed paraffin-embedded sections. HEC-1B endometrial cancer cells treated with VPA showed strong nuclear staining. Control cancer cells from untreated mice had negative or focal weak staining for $\mathrm{p} 21^{\mathrm{WAF} 1}$. This in vivo study shows that VPA at $0.3-1.5 \mathrm{mM}$ inhibited cell proliferation, induced cell cycle arrest and stimulated apoptosis in endometrial cancer cells. This range of concentrations of VPA can be achieved in a patient's serum when that patient is receiving a daily dose of $20-30 \mathrm{mg} / \mathrm{kg}$ for epilepsy. These data are also consistent with the in vitro data. This anticancer activity occurred without any major side-effects, raising hopes that VPA may become a useful therapy for endometrial cancers. Furthermore, VPA has convenient pharmacokinetic properties with a significantly longer biological half-life than the other HDACIs [38].

3.7. MS-275 (Entinostat). MS-275 (MS-27-275) is a synthetic novel benzamide which exerts HDAC inhibitory activity and also induces the expression of the cyclin-dependent kinase inhibitor $\mathrm{p} 21^{\mathrm{WAF} 1}$ and gelsolin, and changes the cell cycle distribution $[38,39]$. MS-275 has shown antiproliferative activity in various in vitro and in vivo human tumor models [40,41], and is currently being tested in clinical trials involving patients with solid tumors or hematological malignancies [42]. The effective dose of MS-275 that inhibited $50 \%$ clonal growth (ED50) of the endometrial cancer cell lines (Ishikawa, HHUA, HEC-1B and RL95-2) was calculated and ranged between $7.8 \times 10^{-7} \mathrm{M}$ and $2.2 \times 10^{-6} \mathrm{M}$ [12] (Table 2). On the other hand, normal endometrial epithelial cells were viable after the treatment with the same doses of MS-275 that induced growth inhibition of endometrial cancer cells [12]. Jiang et al. reported that over the course of 4 days, there was a $60 \%$ reduction in the serous endometrial cancer cell line Ark 2 cell counts by MS-275 (which they called HDAC-I1) $(0.5 \mu \mathrm{M})$ treatments, as compared to controls treated with DMSO solvent (Table 2). They reported growth inhibition of both endometrioid (Ishikawa and AN3) and serous (Ark2) endometrial carcinomas [43].
3.8. M344. Synthetic amide analogs were discovered to have a common structure with TSA [44]. Using an in vitro enzyme inhibition assay of histone deacetylation, Jung et al. demonstrated that M344 is a potent HDACI and an inducer of terminal cell differentiation [44]. The effective dose of M344 that inhibited 50\% clonal growth (ED50) of the Ishikawa endometrial cancer cell line was calculated at $2.3 \times 10^{-6} \mathrm{M}$ [13] (Table 2). On the other hand, normal endometrial epithelial cells were viable after the treatment with the same doses of M344 that induced growth inhibition of endometrial cancer cells [13].

3.9. Apicidin. Cyclic peptide HDACIs can be further divided into two classes: those with an epoxyketone group such as HC-toxin and trapoxin, and those without such a group (apicidin, depsipeptide or FK228). Apicidin is a novel cyclic tetrapeptide with a potent broad spectrum of antiprotozoal activity against Apicomplexan parasites [45]. Its structure is related to trapoxin, a potent HDACI, and some biological activity, including antiproliferative and toxic effects, have been shown in some cancer cell lines [46]. The effective dose of apicidin that inhibited 50\% clonal growth (ED50) of the Ishikawa endometrial cancer cell lines was calculated at $1.0 \times 10^{-6} \mathrm{M}$ [14] (Table 2). On the other hand, normal endometrial epithelial cells were viable after the treatment with the same doses of HDACIs that induced growth inhibition of endometrial cancer cells [14]. Ueda et al. [14] and Ahn et al. [47] independently demonstrated that apicidin has antitumor properties on Ishikawa endometrial cancer cells by selectively inducing the genes related to cell cycle arrest and apoptosis.

3.10. Psammaplin A. Psammaplin A (PsA) is a natural bromotyrosine derivative from a two-sponge association, Poecillastra sp. and Jaspis sp., which was first isolated from the Psammaplysilla sponge [45]. It was reported that PsA has antibacterial and antitumor properties, and also inhibits various enzymes including topoisomerase II, farnesyl protein transferase, leucine amino peptidase, and chitinase (reviewed in [48]). Recently, it was reported that PsA inhibits both HDAC and DNA methyltransferase (DNMT) as epigenetic modifiers of the tumor suppressor gene [49]. PsA caused antiproliferative activity and induced cell cycle arrest or apoptosis in Ishikawa human endometrial cancer cells. PsA inhibited the proliferation of Ishikawa cells in a dose-dependent manner. The $50 \%$ inhibitory concentration (IC50) of PsA was found to be $5 \mu \mathrm{g} / \mathrm{mL}\left(7.5 \times 10^{-6} \mathrm{M}\right)$ after $48 \mathrm{~h}$ treatment (Table 2). PsA increased the proportion in the G1 phase and G2/M phases of the cell cycle [48].

3.11. Oxamflatin. Oxamflatin is an aromatic sulfonamide derivative with a hydroxamic acid group that was identified as a compound inducing the morphological reversion of $\mathrm{v}-\mathrm{K}$ ras-transformed NIH3T3 cells from a chemical library [50]. In addition, the morphology of NIH3T3 cells transformed by various other oncogenes such as v-sis, v-src, $\mathrm{MEK}^{\mathrm{EE}}$ or vfos was also reverted by oxamflatin. Kim et al. analysed the effect of oxamflatin on the proliferation of eight mouse and 
human tumor cell lines. The 50\% inhibitory concentrations of oxamflatin for all the cell lines except CCD-19Lu, a normal human lung cell line, were below $0.72 \mu \mathrm{g} / \mathrm{mL}$, while that for CCD-19Lu was $1.4 \mu \mathrm{g} / \mathrm{mL}$ [51]. Over the course of 4 days, there was a $78 \%$ reduction in the serous endometrial cancer cell line Ark 2 cell counts by oxamflatin $(0.25 \mu \mathrm{M})$ treatments, as compared to controls treated with DMSO solvent. The most striking observation is the $95 \%$ reduction in cell counts following the administration of $0.75 \mu \mathrm{M}$ oxamflatin to Ark2 cells [43]. This report resulted in growth inhibition of both endometrioid (Ishikawa and AN3) and serous (Ark2) endometrial carcinomas.

\section{Conclusions}

In this review, we summarize recent preclinical studies on the use of HDACIs, especially in human endometrial cancer cells. Many questions are currently still unanswered with respect to HDACI specificities for definite tumor subtypes and the molecular mechanisms underlying HDACI-induced differentiation, cell cycle arrest and apoptosis. In addition, the regulation mechanisms of the specific gene expression and recruitment of HDAC complex to the specific promoter sites remain still to be determined. Also, it is still unclear to what extent different HDACs exhibit different and potentially overlapping functions, and it is important to distinguish the HDAC specificity of HDACIs for the development of selective therapy on the molecular level. Further work is needed to improve our understanding of why transformed cells are more susceptible to the effect of HDACIs than normal cells. Also, combinations of HDACIs with differentiation-inducing agents, with cytotoxic agents, and even with gene therapy may represent novel therapeutic strategies and new hope for the treatment of endometrial cancer.

\section{Acknowledgments}

The study was supported by a grant (project code FK344 to NT) from the Japan Society of Gynecologic Oncology, a Grant-in-Aid (no. 21592139 to NT) for Scientific Research from the Ministry of Education, Culture, Sports, Science, and Technology, Japan, and a Research Fund at the Discretion of the President, Oita University.

\section{References}

[1] N. I. Medel, S. Bansal, D. S. Miller, J. D. Wright, and T. J. Herzog, "Pharmacotherapy of endometrial cancer," Expert Opinion on Pharmacotherapy, vol. 10, no. 12, pp. 1939-1951, 2009.

[2] B. D. Strahl and C. D. Allis, "The language of covalent histone modifications," Nature, vol. 403, no. 6765, pp. 41-45, 2000.

[3] T. Jenuwein and C. D. Allis, "Translating the histone code," Science, vol. 293, no. 5532, pp. 1074-1080, 2001.

[4] J. E. Bolden, M. J. Peart, and R. W. Johnstone, "Anticancer activities of histone deacetylase inhibitors," Nature Reviews Drug Discovery, vol. 5, no. 9, pp. 769-784, 2006.
[5] N. Takai and H. Narahara, "Human endometrial and ovarian cancer cells: histone deacetylase inhibitors exhibit antiproliferative activity, potently induce cell cycle arrest, and stimulate apoptosis," Current Medicinal Chemistry, vol. 14, no. 24, pp. 2548-2553, 2007.

[6] M. Haberland, R. L. Montgomery, and E. N. Olson, "The many roles of histone deacetylases in development and physiology: implications for disease and therapy," Nature Reviews Genetics, vol. 10, no. 1, pp. 32-42, 2009.

[7] N. Takai, N. Kawamata, D. Gui, J. W. Said, I. Miyakawa, and H. P. Koeffler, "Human ovarian carcinoma cells: histone deacetylase inhibitors exhibit antiproliferative activity and potently induce apoptosis," Cancer, vol. 101, no. 12, pp. 2760 2770, 2004.

[8] C.-Y. Gui, L. Ngo, W. S. Xu, V. M. Richon, and P. A. Marks, "Histone deacetylase (HDAC) inhibitor activation of p21WAF1 involves changes in promoter-associated proteins, including HDAC1," Proceedings of the National Academy of Sciences of the United States of America, vol. 101, no. 5, pp. 1241-1246, 2004.

[9] N. Takai, J. C. Desmond, T. Kumagai, et al., "Histone deacetylase inhibitors have a profound antigrowth activity in endometrial cancer cells," Clinical Cancer Research, vol. 10, no. 3, pp. 1141-1149, 2004.

[10] N. Takai, T. Ueda, M. Nishida, et al., "CBHA is a family of hybrid polar compounds that inhibit histone deacetylase, and induces growth inhibition, cell cycle arrest and apoptosis in human endometrial and ovarian cancer cells," Oncology, vol. 70, no. 2, pp. 97-105, 2006.

[11] N. Takai, T. Ueda, M. Nishida, K. Nasu, and H. Narahara, "A novel histone deacetylase inhibitor, Scriptaid, induces growth inhibition, cell cycle arrest and apoptosis in human endometrial cancer and ovarian cancer cells," International Journal of Molecular Medicine, vol. 17, no. 2, pp. 323-329, 2006.

[12] N. Takai, T. Ueda, M. Nishida, K. Nasu, and H. Narahara, "Anticancer activity of MS-275, a novel histone deacetylase inhibitor, against human endometrial cancer cells," Anticancer Research, vol. 26, no. 2, pp. 939-945, 2006.

[13] N. Takai, T. Ueda, M. Nishida, K. Nasu, and H. Narahara, "M344 is a novel synthesized histone deacetylase inhibitor that induces growth inhibition, cell cycle arrest, and apoptosis in human endometrial cancer and ovarian cancer cells," Gynecologic Oncology, vol. 101, no. 1, pp. 108-113, 2006.

[14] T. Ueda, N. Takai, M. Nishida, K. Nasu, and H. Narahara, "Apicidin, a novel histone deacetylase inhibitor, has profound anti-growth activity in human endometrial and ovarian cancer cells," International Journal of Molecular Medicine, vol. 19, no. 2, pp. 301-308, 2007.

[15] A. A. Ruefli, M. J. Ausserlechner, D. Bernhard, et al., "The histone deacetylase inhibitor and chemotherapeutic agent suberoylanilide hydroxamic acid (SAHA) induces a cell-death pathway characterized by cleavage of Bid and production of reactive oxygen species," Proceedings of the National Academy of Sciences of the United States of America, vol. 98, no. 19, pp. 10833-10838, 2001.

[16] W. Xu, L. Ngo, G. Perez, M. Dokmanovic, and P. A. Marks, "Intrinsic apoptotic and thioredoxin pathways in human prostate cancer cell response to histone deacetylase inhibitor," Proceedings of the National Academy of Sciences of the United States of America, vol. 103, no. 42, pp. 15540-15545, 2006. 
[17] R. R. Rosato, J. A. Almenara, and S. Grant, "The histone deacetylase inhibitor MS-275 promotes differentiation or apoptosis in human leukemia cells through a process regulated by generation of reactive oxygen species and induction of p21 CIP1/WAF1," Cancer Research, vol. 63, no. 13, pp. 3637-3645, 2003.

[18] D. Marchion and P. Münster, "Development of histone deacetylase inhibitors for cancer treatment," Expert Review of Anticancer Therapy, vol. 7, no. 4, pp. 583-598, 2007.

[19] M. Nishida, "The Ishikawa cells from birth to the present," Human Cell, vol. 15, no. 3, pp. 104-117, 2002.

[20] N. Tsuji, M. Kobayashi, and K. Nagashima, "A new antifungal antibiotic, trichostatin," Journal of Antibiotics, vol. 29, no. 1, pp. 1-6, 1976.

[21] N. Tsuji and M. Kobayashi, "Trichostatin C, a glucopyranosyl hydroxamate," Journal of Antibiotics, vol. 31, no. 10, pp. 939$944,1978$.

[22] V. Sandor, A. R. Robbins, R. Robey, et al., "FR901228 causes mitotic arrest but does not alter microtubule polymerization," Anti-Cancer Drugs, vol. 11, no. 6, pp. 445-454, 2000.

[23] C. Van Lint, S. Emiliani, and E. Verdin, "The expression of a small fraction of cellular genes is changed in response to histone hyperacetylation," Gene Expression, vol. 5, no. 4-5, pp. 245-253, 1996.

[24] S. C. Dowdy, S. Jiang, X. C. Zhou, et al., "Histone deacetylase inhibitors and paclitaxel cause synergistic effects on apoptosis and microtubule stabilization in papillary serous endometrial cancer cells," Molecular Cancer Therapeutics, vol. 5, no. 11, pp. 2767-2776, 2006.

[25] M. S. Finnin, J. R. Donigian, A. Cohen, et al., "Structures of a histone deacetylase homologue bound to the TSA and SAHA inhibitors," Nature, vol. 401, no. 6749, pp. 188-193, 1999.

[26] L. H. Camacho, J. Olson, W. P. Tong, C. W. Young, D. R. Spriggs, and M. G. Malkin, "Phase I dose escalation clinical trial of phenylbutyrate sodium administered twice daily to patients with advanced solid tumors," Investigational New Drugs, vol. 25, no. 2, pp. 131-138, 2007.

[27] S. C. Modesitt, M. Sill, J. S. Hoffman, and D. P. Bender, "A phase II study of vorinostat in the treatment of persistent or recurrent epithelial ovarian or primary peritoneal carcinoma: a Gynecologic Oncology Group study," Gynecologic Oncology, vol. 109, no. 2, pp. 182-186, 2008.

[28] R. Piekarz and S. Bates, "A review of depsipeptide and other histone deacetylase inhibitors in clinical trials," Current Pharmaceutical Design, vol. 10, no. 19, pp. 2289-2298, 2004.

[29] V. M. Richon, S. Emiliani, E. Verdin, et al., "A class of hybrid polar inducers of transformed cell differentiation inhibits histone deacetylases," Proceedings of the National Academy of Sciences of the United States of America, vol. 95, no. 6, pp. 30033007, 1998.

[30] V. M. Richon, Y. Webb, R. Merger, et al., "Second generation hybrid polar compounds are potent inducers of transformed cell differentiation," Proceedings of the National Academy of Sciences of the United States of America, vol. 93, no. 12, pp. 5705-5708, 1996.

[31] G. H. Su, T. A. Sohn, B. Ryu, and S. E. Kern, "A novel histone deacetylase inhibitor identified by high-throughput transcriptional screening of a compound library," Cancer Research, vol. 60, no. 12, pp. 3137-3142, 2000.

[32] J. C. Keen, L. Yan, K. M. Mack, et al., "A novel histone deacetylase inhibitor, scriptaid, enhances expression of functional estrogen receptor alpha (ER) in ER negative human breast cancer cells in combination with 5-aza 2'-deoxycytidine," Breast Cancer Research and Treatment, vol. 81, no. 3, pp. 177186, 2003.

[33] E. P. M. Candido, R. Reeves, and J. R. Davie, "Sodium butyrate inhibits histone deacetylation in cultured cells," Cell, vol. 14, no. 1, pp. 105-113, 1978.

[34] Y. Terao, J.-I. Nishida, S. Horiuchi, et al., "Sodium butyrate induces growth arrest and senescence-like phenotypes in gynecologic cancer cells," International Journal of Cancer, vol. 94, no. 2, pp. 257-267, 2001.

[35] R. P. Warrell Jr., L.-Z. He, V. Richon, E. Calleja, and P. P. Pandolfi, "Therapeutic targeting of transcription in acute promyelocytic leukemia by use of an inhibitor of histone deacetylase," Journal of the National Cancer Institute, vol. 90, no. 21, pp. 1621-1625, 1998.

[36] R. A. Blaheta, H. Nau, M. Michaelis, and J. Cinatl Jr., "Valproate and valproate-analogues: potent tools to fight against cancer," Current Medicinal Chemistry, vol. 9, no. 15, pp. 1417-1433, 2002.

[37] C. J. Phiel, F. Zhang, E. Y. Huang, M. G. Guenther, M. A. Lazar, and P. S. Klein, "Histone deacetylase is a direct target of valproic acid, a potent anticonvulsant, mood stabilizer, and teratogen," The Journal of Biological Chemistry, vol. 276, no. 39, pp. 36734-36741, 2001.

[38] A. Chapman, P. E. Keane, B. S. Meldrum, J. Simiand, and J. C. Vernieres, "Mechanism of anticonvulsant action of valproate," Progress in Neurobiology, vol. 19, no. 4, pp. 315-359, 1982.

[39] T. Suzuki, T. Ando, K. Tsuchiya, et al., "Synthesis and histone deacetylase inhibitory activity of new benzamide derivatives," Journal of Medicinal Chemistry, vol. 42, no. 15, pp. 3001-3003, 1999.

[40] A. Saito, T. Yamashita, Y. Mariko, et al., "A synthetic inhibitor of histone deacetylase, MS-27-275, with marked in vivo antitumor activity against human tumors," Proceedings of the National Academy of Sciences of the United States of America, vol. 96, no. 8, pp. 4592-4597, 1999.

[41] B. I. Lee, S. H. Park, J. W. Kim, et al., "MS-275, a histone deacetylase inhibitor, selectively induces transforming growth factor beta type II receptor expression in human breast cancer cells," Cancer Research, vol. 61, no. 3, pp. 931-934, 2001.

[42] Q. C. Ryan, D. Headlee, M. Acharya, et al., "Phase I and pharmacokinetic study of MS-275, a histone deacetylase inhibitor, in patients with advanced and refractory solid tumors or lymphoma," Journal of Clinical Oncology, vol. 23, no. 17, pp. 3912-3922, 2005.

[43] S. Jiang, S. C. Dowdy, X. W. Meng, et al., "Histone deacetylase inhibitors induce apoptosis in both type I and type II endometrial cancer cells," Gynecologic Oncology, vol. 105, no. 2, pp. 493-500, 2007.

[44] M. Jung, G. Brosch, D. Kölle, H. Scherf, C. Gerhäuser, and P. Loidl, "Amide analogues of trichostatin A as inhibitors of histone deacetylase and inducers of terminal cell differentiation," Journal of Medicinal Chemistry, vol. 42, no. 22, pp. 4669-4679, 1999.

[45] S. J. Darkin-Rattray, A. M. Gurnett, R. W. Myers, et al., "Apicidin: a novel antiprotozoal agent that inhibits parasite histone deacetylase," Proceedings of the National Academy of Sciences of the United States of America, vol. 93, no. 23, pp. 13143-13147, 1996.

[46] J.-W. Han, S. H. Ahn, S. H. Park, et al., "Apicidin, a histone deacetylase inhibitor, inhibits proliferation of tumor cells via induction of p21 $1^{\text {WAF1/Cip1 }}$ and gelsolin," Cancer Research, vol. 60 , no. 21 , pp. $6068-6074,2000$. 
[47] M. Y. Ahn, J. Lee, Y. J. Na, et al., "Mechanism of apicidininduced cell cycle arrest and apoptosis in Ishikawa human endometrial cancer cells," Chemico-Biological Interactions, vol. 179, no. 2-3, pp. 169-177, 2009.

[48] M. Y. Ahn, J. H. Jung, Y. J. Na, and H. S. Kim, "A natural histone deacetylase inhibitor, Psammaplin A, induces cell cycle arrest and apoptosis in human endometrial cancer cells," Gynecologic Oncology, vol. 108, no. 1, pp. 27-33, 2008.

[49] I. C. Pina, J. T. Gautschi, G. Wang, et al., "Psammaplins from the sponge Pseudoceratina purpurea: inhibition of both histone deacetylase and DNA methyltransferase," Journal of Organic Chemistry, vol. 68, no. 10, pp. 3866-3873, 2003.

[50] H. Sonoda, K. Nishida, T. Yoshioka, M. Ohtani, and K. Sugita, "Oxamflatin : a novel compound which reverses malignant phenotype to normal one via induction of JunD," Oncogene, vol. 13, no. 1, pp. 143-149, 1996.

[51] Y. B. Kim, K.-H. Lee, K. Sugita, M. Yoshida, and S. Horinouchi, "Oxamflatin is a novel antitumor compound that inhibits mammalian histone deacetylase," Oncogene, vol. 18, no. 15, pp. 2461-2470, 1999. 


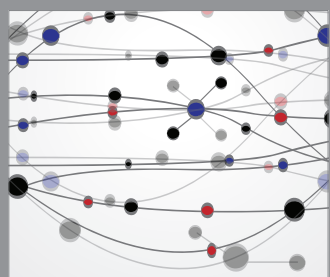

The Scientific World Journal
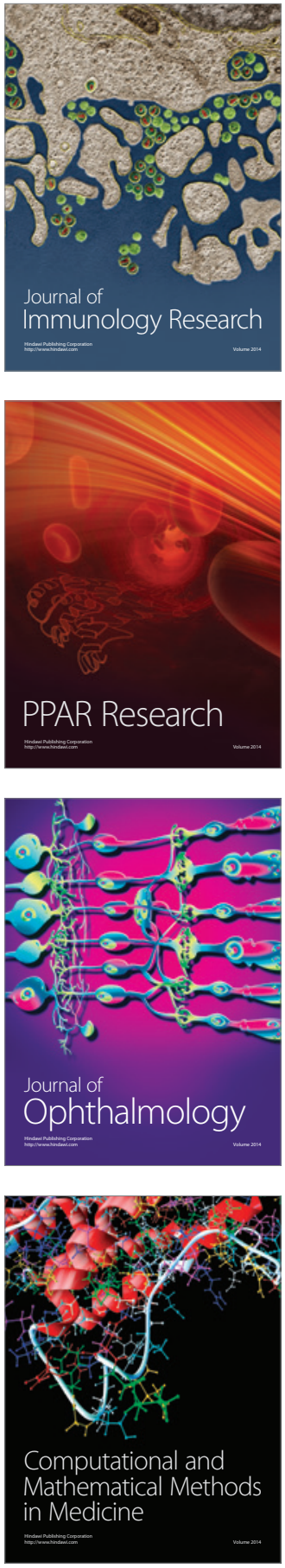

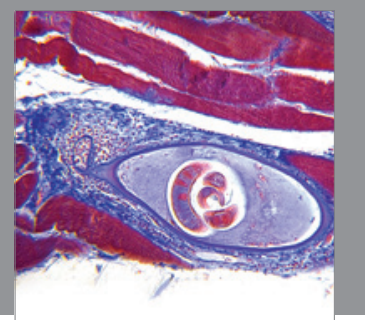

Gastroenterology

Research and Practice
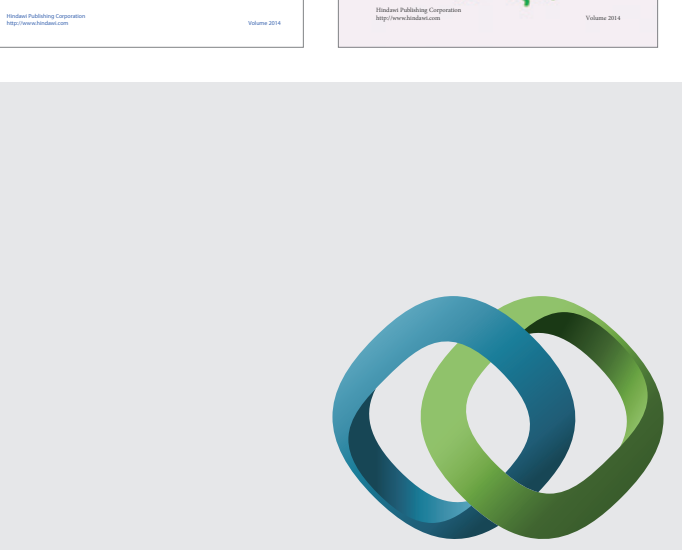

\section{Hindawi}

Submit your manuscripts at

http://www.hindawi.com
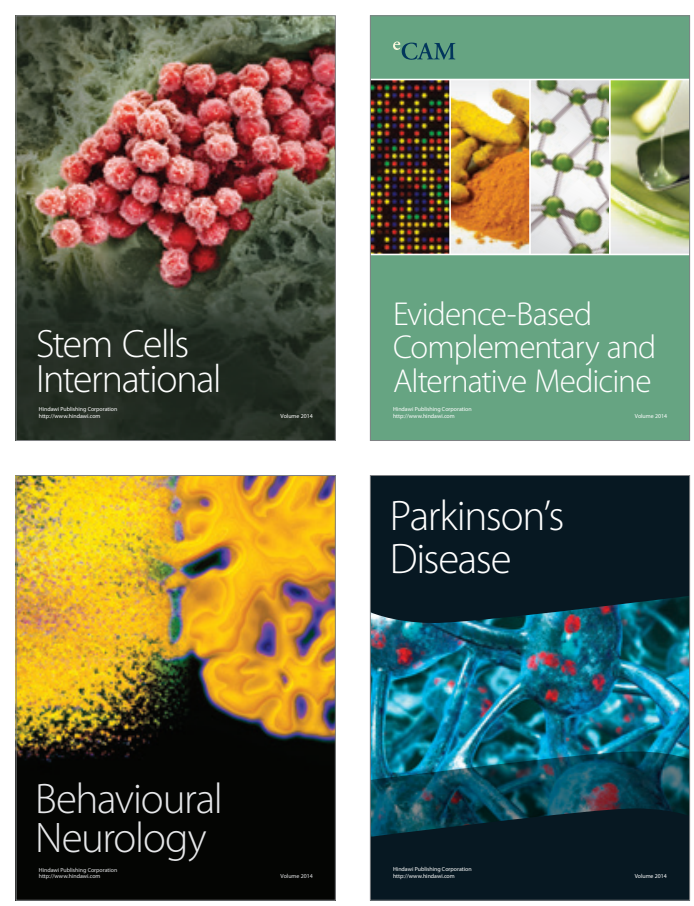

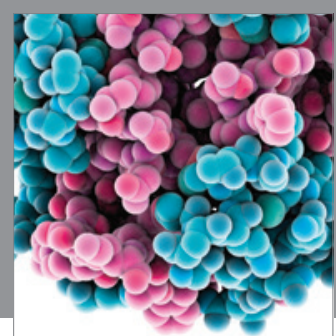

Journal of
Diabetes Research



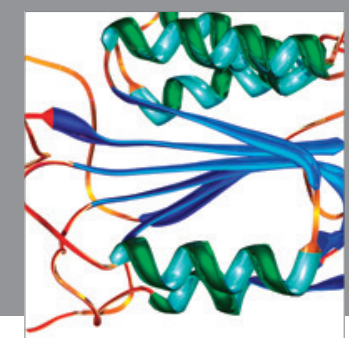

Disease Markers
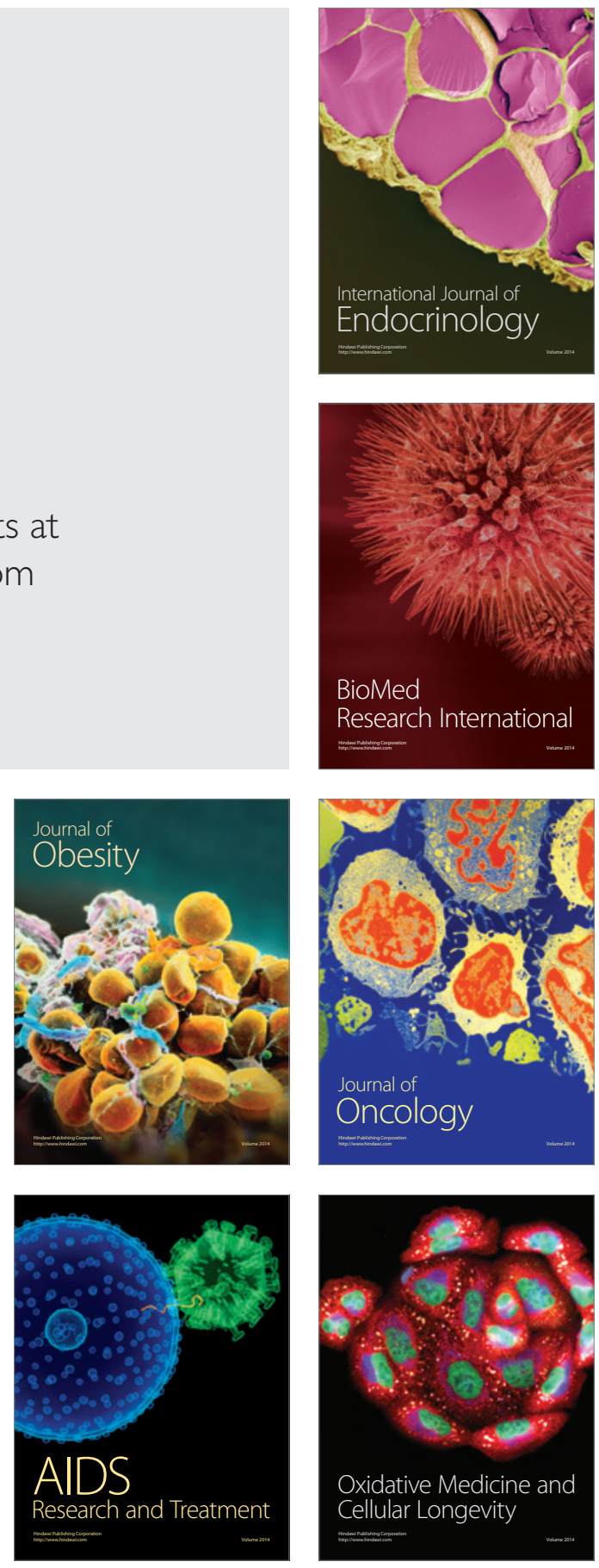